Document downloaded from:

http://hdl.handle.net/10251/172302

This paper must be cited as:

Del Campo, R.; Fernández, A.; Mayoral, F.; Naranjo, F.; Sánchez Pérez, EA. (2020). Lorentz spaces of vector measures and real interpolation of operators. Quaestiones Mathematicae. 43(5-6):591-609. https://doi.org/10.2989/16073606.2019.1605413

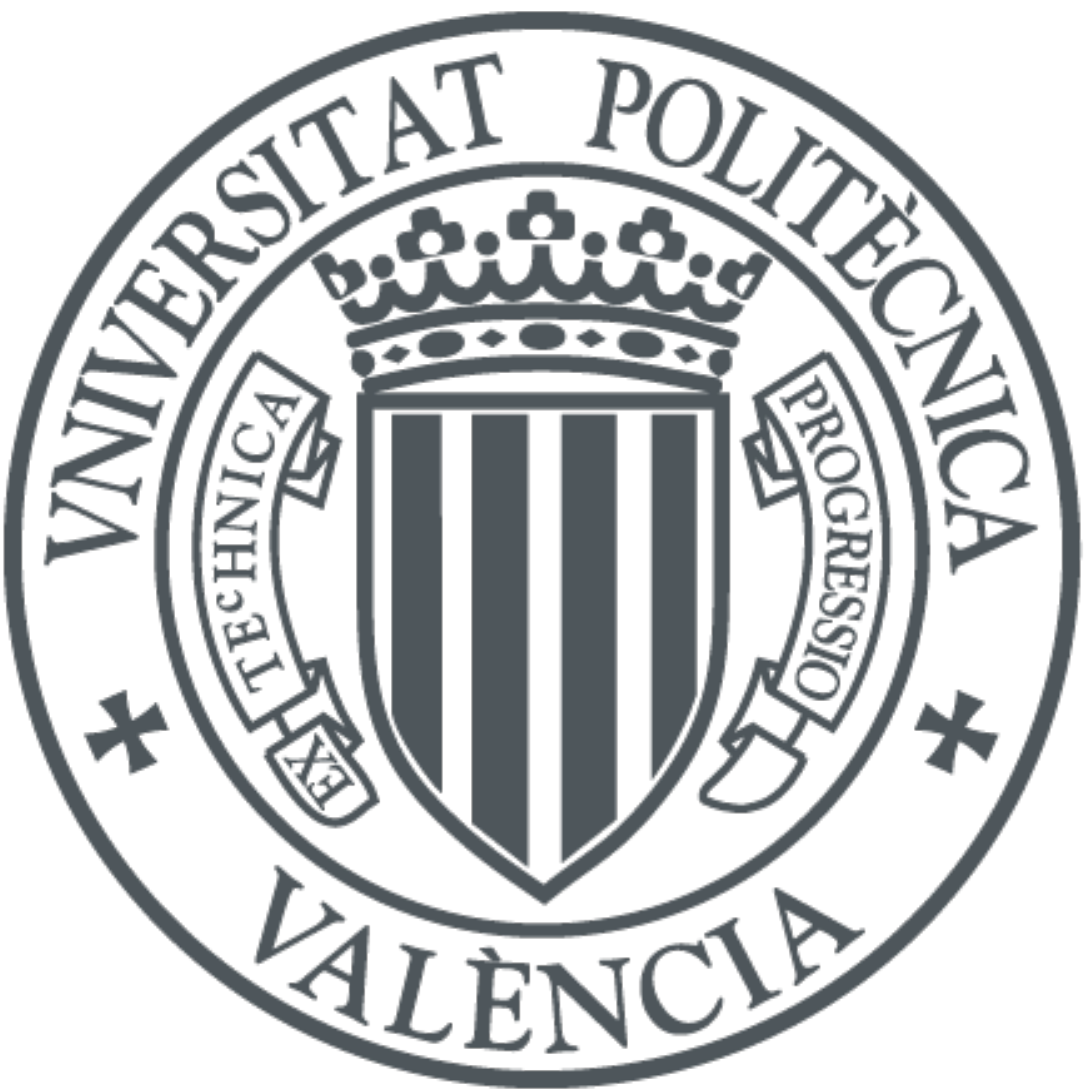

The final publication is available at

https://doi.org/10.2989/16073606.2019.1605413

Copyright Informa UK (National Inquiry Services Center)

Additional Information 


\title{
LORENTZ SPACES OF VECTOR MEASURES AND REAL INTERPOLATION OF OPERATORS
}

\author{
R. DEL CAMPO, A. FERNÁNDEZ, F. MAYORAL, F. NARANJO, E.A. SÁNCHEZ PÉREZ
}

Dedicated to the memory of Joe Diestel

\begin{abstract}
Using the representation of the real interpolation of spaces of $p$-integrable functions with respect to a vector measure, we show new factorization theorems for $p$-th power factorable operators acting in interpolation couples of Banach function spaces. The recently introduced Lorentz spaces of the semivariation of vector measures play a central role in the resulting factorization theorems. We apply our results to analyze extension of operators from classical weighted Lebesgue $L^{p}$-spaces -in general with different weights-, that can be extended to their $q$-th powers. This is the case, for example, of the convolution operators defined by $L^{p}$-improving measures acting in Lebesgue $L^{p}$-spaces or Lorentz spaces. A new representation theorem for Banach lattices with a special lattice geometric property, as a space of vector measure integrable functions, is also proved.
\end{abstract}

\section{Introduction}

The interpolation of operators acting in Banach function spaces has become a classic tool in mathematical analysis, and many important theorems have been obtained in functional analysis using interpolation techniques. Concerning real interpolation of Banach spaces, some relevant results involving operators acting in the classical Lebesgue $L^{p}$-spaces have been extended to their corresponding natural class of real interpolation spaces: the Lorentz spaces. In this paper, the vector measure version of the $L^{p}$-spaces and the associated class of operators factoring through them are considered. Some effort has been made in recent years to improve the knowledge on these spaces, concretely to investigate the role of the $L^{p}$ spaces of a vector measure in the factorization theorems for operators, in comparison with the one played by the classical Lebesgue spaces, mainly in the so-called Maurey-Rosenthal factorization theorems (see [7]).

In the vector valued context and after the work developed by several authors (see $[20,12]$ and the references therein) we learned that the canonical operators that factor through an $L^{p}$-space of a vector measure coincide with the operators that can be extended to the $p$-th power of the Banach function space where the operator acts. These operators are called $p$-th power factorable operators. For example, for $1 \leq p \leq r$ an operator $T: L^{r}[0,1] \longrightarrow E$ is $p$-th power factorable if it can be extended to an operator $\hat{T}$ : $L^{\frac{r}{p}}[0,1] \longrightarrow E$.

Date: February 13, 2021.

2000 Mathematics Subject Classification. Primary 46E30; Secondary 47B38, 46B42.

Key words and phrases. Banach function space, vector measure, real interpolation, factorable operator, bidual concave operator, improving measures.

The first four authors acknowledge the support of La Junta de Andalucía (Spain). The fifth author acknowledges the support of the Ministerio de Economía y Competitividad (Spain) and FEDER under grant MTM2016-77054-C2-1-P. 
Consequently, the analysis of the vector valued version of these classical factorization results is the study of the real interpolation of $p$-th power factorable operators. That is, to analyze to what extent the analogies from the classical setting of interpolation of operators and factorizations through Lorentz spaces can be translated into the framework of spaces of vector measure integrable functions. Motivated by the extension of some classical interpolation results to the vector measure context, we will provide new factorization theorems through a new class of (quasi-)Banach function spaces. As an application, we will use them to produce some new results on factorization and extensions of operators acting on real interpolation spaces of classical Lebesgue $L^{p}$ and Lorentz spaces with different weights.

Regarding the available tools, a concrete representation of the real interpolation spaces of the spaces of $p$-integrable functions with respect to a vector measure has been recently obtained (see [9]). The new relevant Lorentz space $L^{p, q}(\|m\|)$ appears in it. This space is defined following the classical construction of the Lorentz space, but the distribution function is defined by the (real valued) capacity $\|m\|$, - the semivariation of the vector measure $m$-. Since these spaces are in fact the cornerstone of our construction, we will give more results on their geometric properties, and a characterization of what kind of Banach lattices can be represented as Lorentz spaces of the semivariation of a vector measure. This will provide a new representation theorem for this particular class of Banach lattices, which is one of the main outcomes of the present paper.

\section{PRELIMINARIES AND NOTATION}

Let $(\Omega, \Sigma, \mu)$ be a finite measure space. A Banach function space $X$ over $\mu$ (B.f.s. for short) is an ideal of the space of (equivalence classes of) measurable functions $L^{0}(\mu)$ endowed with a complete norm $\|\cdot\|_{X}$ that is compatible with the $\mu$-a.e. order and such that $L^{\infty}(\mu) \subseteq X \subseteq L^{1}(\mu)$ (see [17, p. 28]). The topological dual is denoted by $X^{*}$. A B.f.s. $X$ is order continuous if for every sequence $\left(f_{n}\right)_{n}$ in $X$ such that $0 \leq f_{n} \downarrow 0$ pointwise we have that $\left\|f_{n}\right\|_{X} \downarrow 0$. Let us show in this section several analytical concepts and tools that will be used in the paper.

2.1. $\mathbf{L}^{\mathbf{P}}$-spaces of a vector measure. Let $(\Omega, \Sigma)$ be a measurable space and $E$ a real Banach space. Let $m: \Sigma \longrightarrow E$ be a countably additive vector measure. For every $x^{*}$ in the dual $E^{*}$ of $E$, let $\left\langle m, x^{*}\right\rangle$ be the scalar signed measure defined by $\left\langle m, x^{*}\right\rangle(A):=$ $\left\langle m(A), x^{*}\right\rangle$, for all $A \in \Sigma$. The semivariation of $m$ is the subadditive real bounded set function $\|m\|: A \in \Sigma \longrightarrow\|m\|(A) \in[0, \infty)$ defined by

$$
\|m\|(A)=\sup \left\{\left|\left\langle m, x^{*}\right\rangle\right|(A):\left\|x^{*}\right\|_{E^{*}} \leq 1\right\}
$$

where $\left|\left\langle m, x^{*}\right\rangle\right|$ is the variation measure of $\left\langle m, x^{*}\right\rangle$. It is well-known that

$$
\frac{1}{2}\|m\|(A) \leq \sup \left\{\|m(B)\|_{E}: B \subseteq A, B \in \Sigma\right\} \leq\|m\|(A),
$$

for every set $A \in \Sigma$. A Rybakov (control) measure for $m$ is a measure defined as $\left|\left\langle m, x^{*}\right\rangle\right|$ for some $x^{*} \in E^{*}$, satisfying that $\left|\left\langle m, x^{*}\right\rangle\right|(A)=0$ if and only if $\|m\|(A)=0$. Such a measure always exists (see [8, Theorem IX.2.2]).

Let us introduce now the basic notions of integration with respect to a vector measure. The reader can find a complete analysis of these spaces in [20, Chapter 3]. A measurable function $f: \Omega \longrightarrow \mathbb{R}$ is scalarly integrable if $f \in L^{1}\left(\left|\left\langle m, x^{*}\right\rangle\right|\right)$ for all $x^{*} \in E^{*}$. The space consisting of all (equivalence classes of $\|m\|$-a.e. equal) scalarly integrable functions with respect to $m$ is $L_{w}^{1}(m)$, which is a Banach function space over every Rybakov (control) 
measure for $m$, with the sequential Fatou property, and a weak unit, when equipped with the norm

$$
\|f\|_{L_{w}^{1}(m)}:=\sup \left\{\int_{\Omega}|f| d\left|\left\langle m, x^{*}\right\rangle\right|:\left\|x^{*}\right\|_{E^{*}} \leq 1\right\} .
$$

A function $f \in L_{w}^{1}(m)$ is said to be integrable with respect to $m$ if for every $A \in \Sigma$ there exists an element of $E$ denoted by $\int_{A} f d m$, such that

$$
\left\langle\int_{A} f d m, x^{*}\right\rangle=\int_{A} f d\left\langle m, x^{*}\right\rangle, \quad x^{*} \in E^{*} .
$$

The space of all ( $\|m\|$-a.e. equivalence classes of) integrable functions with the norm of $L_{w}^{1}(m)$ is denoted by $L^{1}(m)$. It is an order continuous ideal of $L_{w}^{1}(m)$, and the integration $\operatorname{map} I_{m}: f \in L^{1}(m) \longrightarrow I_{m}(f):=\int_{\Omega} f d m \in E$ is linear and continuous. The definition of the corresponding $L^{p}(m)$ spaces $(1<p<\infty)$ is done in the natural way. A function $f \in L_{w}^{1}(m)$ is called scalarly $p$-integrable with respect to $m$ if $|f|^{p} \in L_{w}^{1}(m)$, and $p$ integrable with respect to $m$ whenever $|f|^{p} \in L^{1}(m)$. We denote by $L_{w}^{p}(m)$ and $L^{p}(m)$ the corresponding spaces of ( $\|m\|$-a.e. equal equivalence classes of ) scalarly $p$-integrable and $p$-integrable functions with respect to $m$. These spaces are equipped with the norm

$$
\|f\|_{L_{w}^{p}(m)}=\sup \left\{\left(\int_{\Omega}|f|^{p} d\left|\left\langle m, x^{*}\right\rangle\right|\right)^{\frac{1}{p}}:\left\|x^{*}\right\|_{E^{*}} \leq 1\right\} .
$$

Both spaces are $p$-convex (for the definition of $p$-convexity of Banach lattices see [17, 1.d.3]). Finally $L^{\infty}(m)$ denotes the Banach function space of the ( $\|m\|$-a.e. equal equivalence classes of) measurable functions that are $\|m\|$-a.e. essentially bounded.

2.2. Lorentz spaces of the semivariation of a vector measure. Let us present here some fundamental known properties of the Lorentz space of the semivariation of a vector measure $m$. The reader can find a complete explanation of these properties in $[9$, Section 4]. Suppose that $1 \leq p, q \leq \infty$. The Lorentz space $L^{p, q}(\|m\|)$ is defined by all $(\|m\|$-a.e. equivalence classes of) measurable functions $f: \Omega \longrightarrow \mathbb{R}$ for which

$$
\|f\|_{L^{p, q}(\|m\|)}:=\left(\int_{0}^{\infty}\left(s^{\frac{1}{p}} f_{*}(s)\right)^{q} \frac{d s}{s}\right)^{\frac{1}{q}}<\infty, \quad 1 \leq q<\infty,
$$

or $\|f\|_{L^{p, \infty}(\|m\|)}:=\sup \left\{s^{\frac{1}{p}} f_{*}(s): s>0\right\}<\infty$, for $q=\infty$. In these formulas, $f_{*}$ is the decreasing rearrangement of the function $f$ with respect to the semivariation $\|m\|$. These functions (2.1) provide quasi-norms on the corresponding spaces $L^{p, q}(\|m\|)$, but they are equivalent to a norm for $p>1$ and $1 \leq q \leq \infty$. In this case, more can be said: $L^{p, q}(\|m\|)$ is a Banach lattice, that is reflexive (and then order continuous) if $1<p, q<\infty$ (see $[9$, Corollary 14] and [9, Corollary 18]). Moreover, [9, Proposition 2] provides the following equivalent formula for the quasi-norm of the Lorentz spaces. For $1 \leq p, q<\infty$,

$$
\|f\|_{L^{p, q}(\|m\|)}=\left(p \int_{0}^{\infty} t^{q-1}\left(\|m\|_{f}(t)\right)^{\frac{q}{p}} d t\right)^{\frac{1}{q}}
$$

and $\|f\|_{L^{p, \infty}(\|m\|)}=\sup \left\{t\|m\|_{f}(t)^{\frac{1}{p}}: t>0\right\}$, for the case $q=\infty$. Here $\|m\|_{f}$, defined for $t>0$ by the equality $\|m\|_{f}(t):=\|m\|([|f|>t])$, is the distribution function of the function $f$ with respect to the semivariation $\|m\|$, where $[|f|>t]$ denotes the measurable set $\{w \in \Omega:|f(w)|>t\}$. 
For a given vector measure $m$, the inclusions between the different Lorentz spaces $L^{p, q}(\|m\|)$ follow the same rule that for the case of finite scalar measures. In particular $L^{p, q}(\|m\|) \subseteq L^{1}(m)$ for every $1<p<\infty$ and $1 \leq q \leq \infty$. However, the following inclusions are characteristic of the vector valued measure case (see [9, Proposition 7]). For $1 \leq p<\infty$, we have the continuous inclusions

$$
L^{p, 1}(\|m\|) \subseteq L^{p, p}(\|m\|) \subseteq L^{p}(m) \subseteq L_{w}^{p}(m) \subseteq L^{p, \infty}(\|m\|) .
$$

To finish this summary, let us write the main real interpolation result for $L^{p}$-spaces of a vector measure $m$ (see [9, Corollary 17]), which is the main tool for much of this paper.

Theorem 2.1. Suppose $0<\theta<1 \leq q \leq \infty$ and let $1 \leq p_{0} \neq p_{1} \leq \infty$. Then

$$
\left(L^{p_{0}}(m), L^{p_{1}}(m)\right)_{\theta, q}=\left(L_{w}^{p_{0}}(m), L^{p_{1}}(m)\right)_{\theta, q}=\left(L_{w}^{p_{0}}(m), L_{w}^{p_{1}}(m)\right)_{\theta, q}=L^{p, q}(\|m\|),
$$

where $\frac{1}{p}=\frac{1-\theta}{p_{0}}+\frac{\theta}{p_{1}}$. All above equalities are topological.

\section{REAL INTERPolation AND OPTIMAL DOMAins}

Let $T: X \longrightarrow E$ be a Banach space valued operator acting in an order continuous B.f.s. $X$. The expression $m_{T}(A):=T\left(\chi_{A}\right)$ defines a vector measure $m_{T}: \Sigma \longrightarrow E$ which is called the vector measure associated to $T$. The operator $T$ is said to be $\mu$-determined if the measures $\mu$ and $m_{T}$ have exactly the same null sets. When $T$ is $\mu$-determined, the space $L^{1}\left(m_{T}\right)$ is an order continuous Banach function lattice on $(\Omega, \Sigma, \mu), X$ is continuously included into $L^{1}\left(m_{T}\right)$ via the natural inclusion

$$
J_{T}: f \in X \longrightarrow J_{T}(f):=f \in L^{1}\left(m_{T}\right)
$$

and the integration operator $I_{m_{T}}: L^{1}\left(m_{T}\right) \longrightarrow E$ is the unique continuous linear extension of $T$ satisfying $T=I_{m_{T}} \circ J_{T}$ (see [5] or [20, Proposition 4.4]). Therefore, if $Y$ is another order continuous B.f.s such that $X \subseteq Y \subseteq L^{0}(\mu)$ and $T: Y \longrightarrow E$ is a continuous linear extension of $T$, then $Y \subseteq L^{1}\left(m_{T}\right)$ continuously. In this sense, it is said that $L^{1}\left(m_{T}\right)$ is the (order continuous) optimal domain for the operator $T$.

Let us consider now an interpolation couple $\left(X_{0}, X_{1}\right)$ of order continuous Banach function spaces on the same finite measure space $(\Omega, \Sigma, \mu)$, and an interpolation couple $\left(E_{0}, E_{1}\right)$ of Banach spaces. Take and admissible $\mu$-determined operator $T$ between the couples $\left(X_{0}, X_{1}\right)$ and $\left(E_{0}, E_{1}\right)$, that is, an operator $T: X_{0}+X_{1} \longrightarrow E_{0}+E_{1}$ such that its restrictions $T_{0}:=\left.T\right|_{X_{0}}: X_{0} \longrightarrow E_{0}$ and $T_{1}:=\left.T\right|_{X_{1}}: X_{1} \longrightarrow E_{1}$ are continuous. Moreover, let $T_{\theta, q}:\left(X_{0}, X_{1}\right)_{\theta, q} \longrightarrow\left(E_{0}, E_{1}\right)_{\theta, q}$ be the interpolated operator for $0<\theta<$ $1 \leq q<\infty$, where $(\cdot, \cdot)_{\theta, q}$ denotes the real interpolation method, and set $m_{\theta, q}:=m_{T_{\theta, q}}$ for all $0<\theta<1 \leq q<\infty$. In this situation we have the optimal domains $L^{1}\left(m_{0}\right), L^{1}\left(m_{1}\right)$, and $L^{1}\left(m_{\theta, q}\right)$ corresponding to the restricted $\mu$-determined operators $T_{0}: X_{0} \longrightarrow E_{0}$, $T_{1}: X_{1} \longrightarrow E_{1}$ and $T_{\theta, q}:\left(X_{0}, X_{1}\right)_{\theta, q} \longrightarrow\left(E_{0}, E_{1}\right)_{\theta, q}$. The following result relates the real interpolation space $\left(L^{1}\left(m_{0}\right), L^{1}\left(m_{1}\right)\right)_{\theta, q}$ of the optimal domains of $T_{0}$ and $T_{1}$ with the optimal domain $L^{1}\left(m_{\theta, q}\right)$ of the interpolated operator $T_{\theta, q}$. The case of the complex interpolation method was considered in [2, Theorem 3.1].

Theorem 3.1. If $0<\theta<1 \leq q<\infty$, then $\left(L^{1}\left(m_{0}\right), L^{1}\left(m_{1}\right)\right)_{\theta, q} \subseteq L^{1}\left(m_{\theta, q}\right)$. Moreover, this inclusion is continuous.

Proof. For $i=0,1$, the space $X_{i}$ is continuously included into $L^{1}\left(m_{i}\right)$, and there exists a unique extension of $T_{i}$ to $L^{1}\left(m_{i}\right)$ given by the integration map $I_{m_{i}}: L^{1}\left(m_{i}\right) \longrightarrow E_{i}$ (see 
[5] or [20, Theorem 4.14]). Since $I_{m_{0}}(\varphi)=I_{m_{1}}(\varphi)$ for every simple function $\varphi$ and the set of simple functions are dense in both spaces $L^{1}\left(m_{0}\right)$ and $L^{1}\left(m_{1}\right)$, the map

$$
\hat{T}: L^{1}\left(m_{0}\right)+L^{1}\left(m_{1}\right) \longrightarrow E_{0}+E_{1}
$$

given by $\hat{T}(f):=I_{m_{0}}\left(f_{0}\right)+I_{m_{1}}\left(f_{1}\right)$ for $f_{0} \in L^{1}\left(m_{0}\right), f_{1} \in L^{1}\left(m_{1}\right)$ is well-defined, linear and continuous, that is, it is an operator.

The interpolated spaces $\left(X_{0}, X_{1}\right)_{\theta, q}$ and $\left(L^{1}\left(m_{0}\right), L^{1}\left(m_{1}\right)\right)_{\theta, q}$ are both order continuous, since $X_{0}, X_{1}, L^{1}\left(m_{0}\right)$ and $L^{1}\left(m_{1}\right)$ are all order continuous (see Remarks 1.9 and 1.10 in [6, page 17]). The simple functions are also dense in both interpolated spaces since they are dense in each intersection, $X_{0} \cap X_{1}$ and $L^{1}\left(m_{0}\right) \cap L^{1}\left(m_{1}\right)$. Moreover, the following inclusion $\left(X_{0}, X_{1}\right)_{\theta, q} \subseteq\left(L^{1}\left(m_{0}\right), L^{1}\left(m_{1}\right)\right)_{\theta, q}$ holds. The restriction of the interpolated operator $\hat{T}_{\theta, q}:\left(L^{1}\left(m_{0}\right), L^{1}\left(m_{1}\right)\right)_{\theta, q} \longrightarrow\left(E_{0}, E_{1}\right)_{\theta, q}$ to the space $\left(X_{0}, X_{1}\right)_{\theta, q}$ clearly coincides with $T_{\theta, q}$. In other words, $\hat{T}_{\theta, q}$ is a continuous linear extension of $T_{\theta, q}$ to the order continuous Banach function space $\left(L^{1}\left(m_{0}\right), L^{1}\left(m_{1}\right)\right)_{\theta, q}$. The optimality of the domain $L^{1}\left(m_{\theta, q}\right)$ for $T_{\theta, q}$ (see again [5] or [20, Theorem 4.14]) gives the continuous inclusion $\left(L^{1}\left(m_{0}\right), L^{1}\left(m_{1}\right)\right)_{\theta, q} \subseteq L^{1}\left(m_{\theta, q}\right)$.

Remark 3.2. In general the inclusion $\left(L^{1}\left(m_{0}\right), L^{1}\left(m_{1}\right)\right)_{\theta, q} \subseteq L^{1}\left(m_{\theta, q}\right)$ is proper. The example considered in [2, Remark 3.3] for the complex interpolation method, also works in this case.

Next result is a consequence of the above theorem combined with the estimates of the $K$-functional of $p$-convexifications of Banach function spaces obtained by Maligranda in [19, Lemma 1 and Theorem 1].

Corollary 3.3. If $0<\theta<1 \leq q<\infty$, and $1 \leq p<\infty$, then

$$
\left(L^{p}\left(m_{0}\right), L^{p}\left(m_{1}\right)\right)_{\theta, q} \subseteq L^{p}\left(m_{\theta, q}\right) .
$$

Proof. Since $q \leq p q$, we have $\left(L^{p}\left(m_{0}\right), L^{p}\left(m_{1}\right)\right)_{\theta, q} \subseteq\left(L^{p}\left(m_{0}\right), L^{p}\left(m_{1}\right)\right)_{\theta, p q}$. Then it is enough to prove that $\left(L^{p}\left(m_{0}\right), L^{p}\left(m_{1}\right)\right)_{\theta, p q} \subseteq L^{p}\left(m_{\theta, q}\right)$. We have to see that $|f|^{p} \in$ $L^{1}\left(m_{\theta, q}\right)$ for a given $f \in\left(L^{p}\left(m_{0}\right), L^{p}\left(m_{1}\right)\right)_{\theta, p q}$. By Theorem 3.1 we need to prove $|f|^{p} \in$ $\left(L^{1}\left(m_{0}\right), L^{1}\left(m_{1}\right)\right)_{\theta, q}$. In order to obtain that, we use Maligranda's estimates. If $f \in$ $\left(L^{p}\left(m_{0}\right), L^{p}\left(m_{1}\right)\right)_{\theta, p q}$, then

$$
\begin{aligned}
\int_{0}^{\infty}\left(\frac{K\left(t,|f|^{p}, L^{1}\left(m_{0}\right), L^{1}\left(m_{1}\right)\right)}{t^{\theta}}\right)^{q} \frac{d t}{t} & =p \int_{0}^{\infty}\left(\frac{K\left(s^{p},|f|^{p}, L^{1}\left(m_{0}\right), L^{1}\left(m_{1}\right)\right)}{s^{p \theta}}\right)^{q} \frac{d s}{s} \\
\text { by [19, Theorem 1] } & \approx \int_{0}^{\infty}\left(\frac{K\left(s,|f|, L^{p}\left(m_{0}\right), L^{p}\left(m_{1}\right)\right)^{p}}{s^{p \theta}}\right)^{q} \frac{d s}{s} \\
& =\int_{0}^{\infty}\left(\frac{K\left(s,|f|, L^{p}\left(m_{0}\right), L^{p}\left(m_{1}\right)\right)}{s^{\theta}}\right)^{p q} \frac{d s}{s}
\end{aligned}
$$

The last integral is finite, and so the proof is over.

\section{Real interpolation of P-TH POWER FaCtorable OpERATORS}

Let us start with the real interpolated factorization theorems for $p$-th power factorable operators. Let $X$ be an order continuous Banach function space over a finite measure $\mu$ and $T: X \longrightarrow E$ an operator on a Banach space $E$. The formula $m_{T}(A):=T\left(\chi_{A}\right)$ provides a (countably additive) vector measure in $E$ associated to the operator $T$. In order 
to avoid some technicalities, throughout the paper, every operator $T$ will be assumed to be $\mu$-determined whenever a factorization is established, although in most of the results this property is not needed.

As we said in the Introduction, the class of operators that factor through spaces $L^{p}\left(m_{T}\right)$ (for $\left.1 \leq p<\infty\right)$ is the class of the so called $p$-th power factorable operators (see $[20$, Chapter 5]). Recall that an operator $T: X \longrightarrow E$ is said to be $p$-th power factorable if there is a constant $K>0$ such that

$$
\|T(f)\|_{E} \leq K\left\||f|^{\frac{1}{p}}\right\|_{X}^{p}, \quad f \in X .
$$

The expression (without constant) on the right hand side of inequality (4.1) is in fact the quasi-norm on the space $X_{[p]}$ defined as the space of all $f$ such that $|f|^{\frac{1}{p}} \in X$. It is well-known that $X \subseteq X_{[p]}$ (see [20, Chapter 2]), and so it means that the requirement given by the inequality (4.1) is more restrictive as $p$ increases. It is well-known that an operator $T$ is $p$-th power factorable if and only if it factors through $L^{p}\left(m_{T}\right)$ as

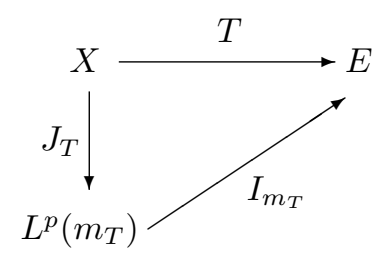

See $[20$, Chapter 5] and also $[2,13,14]$ for general information on these operators. Our aim is to analyze which additional information can be obtained by the factorization of the operators through the corresponding Lorentz spaces of their semivariations, that is obtained in a natural way when two $p$-th power factorable operators are interpolated (see [2] for some related results for the complex interpolation method). Our main results provide factorizations through well described Banach function spaces, that are the corresponding Lorentz spaces of their semivariations.

A consequence of Corollary 3.3 is that the real interpolated operator of two $p$-th power factorable operators is also $p$-th power factorable.

Corollary 4.1. If $T_{0}$ and $T_{1}$ are $p$-th power factorable for some $1<p<\infty$, then $T_{\theta, q}$ is $p$-th power factorable for each $0<\theta<1 \leq q<\infty$.

Proof. Recall that an operator $T: X \longrightarrow E$ is $p$-th power factorable if and only if $X \subseteq$ $L^{p}\left(m_{T}\right)$. Then it is enough to check that $\left(X_{0}, X_{1}\right)_{\theta, q} \subseteq L^{p}\left(m_{\theta, q}\right)$. But this is clear taking into account that $T_{0}$ and $T_{1}$ are $p$-th power factorable by applying Corollary 3.3.

Remark 4.2. Suppose as in the previous corollary that the operators $T_{0}$ and $T_{1}$ are both $p$-th power factorable for some $1<p<\infty$. Since $L^{p}\left(m_{\theta, q}\right) \subseteq L^{r, q}\left(\left\|m_{\theta, q}\right\|\right)$ for all $1<r<p$ and all $1 \leq q<\infty$, we obtain the following commutative diagram

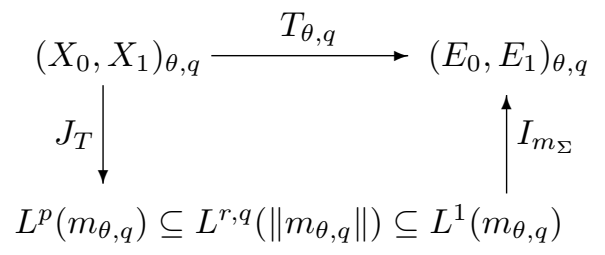


Here $m_{\Sigma}$ denotes the measure associated to the operator $T$ with values into the sum space $E_{0}+E_{1}$. Note that the restriction of the integration operator $I_{m_{\Sigma}}: L^{1}\left(m_{\Sigma}\right) \longrightarrow E_{0}+E_{1}$ to each $L^{1}\left(m_{\theta, q}\right)$ is just the integration operator $I_{m_{\theta, q}}: L^{1}\left(m_{\theta, q}\right) \longrightarrow\left(E_{0}, E_{1}\right)_{\theta, q}$.

Let us consider now the general situation. It is given by the case in which $T_{0}$ is $p_{0}$-th power factorable and $T_{1}$ is $p_{1}$-th power factorable. For the aim of simplicity, we will assume in this case that the range space is the same for both operators. That is, we have a $\mu$-determined operator $T: X_{0}+X_{1} \longrightarrow E$. Note that we have only one vector measure

$$
m: A \in \Sigma \longrightarrow m(A):=T\left(\chi_{A}\right) \in E
$$

associated to every restriction of $T$ to any order continuous Banach function space included into the sum $X_{0}+X_{1}$. The following result can be obtained by using direct arguments of real interpolation of function spaces together with Theorem 2.1.

Theorem 4.3. Let $1 \leq p_{0} \neq p_{1}<\infty$. If $T_{0}$ is $p_{0}$-th power factorable and $T_{1}$ is $p_{1}$-th power factorable, then for each $0<\theta<1 \leq q<\infty$, the interpolated operator $T_{\theta, q}$ factors through the following commutative diagram

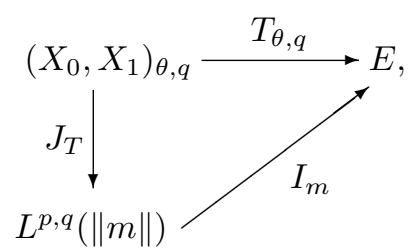

where $p>1$ is given by $\frac{1}{p}=\frac{1-\theta}{p_{0}}+\frac{\theta}{p_{1}}$.

Proof. Using the hypothesis, we directly obtain the continuous inclusions $X_{k} \subseteq L^{p_{k}}(m)$ for $k=0,1$. Then we get that $\left(X_{0}, X_{1}\right)_{\theta, q} \subseteq\left(L^{p_{0}}(m), L^{p_{1}}(m)\right)_{\theta, q}$ for all $0<\theta<1 \leq q<$ $\infty$. Now, Theorem 2.1 gives $\left(L^{p_{0}}(m), L^{p_{1}}(m)\right)_{\theta, q}=L^{p, q}(\|m\|)$, where $\frac{1}{p}=\frac{1-\theta}{p_{0}}+\frac{\theta}{p_{1}}$, so we obtain the commutative diagram

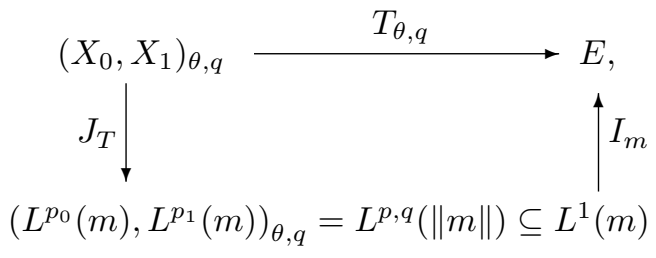

and the proof is over.

Remark 4.4. Since $p_{0} \neq p_{1}$, we can assume for example that $p_{0}<p_{1}$. Then $L^{p_{1}}(m) \subseteq$ $L^{p, q}(\|m\|) \subseteq L^{p_{0}}(m)$. Thus, as a consequence of Theorem 4.3 we obtain a factorization for the interpolated operator $T_{\theta, q}$ that is weaker than being $p_{1}$-th power factorable but stronger than being $p_{0}$-th power factorable. In the next section we will look specifically at operators with this property. 


\section{LORENTZ FACTORABLE OPERATORS}

Motivated by the results of the previous section, we say that an operator $T: X \longrightarrow E$ with values on a Banach space $E$ and acting in an order continuous Banach function space $X$ over a finite measure $\mu$ is $(p, q)$-Lorentz factorable, with $1<p<\infty$, and $1 \leq q<\infty$, if it factors through the following commutative diagram

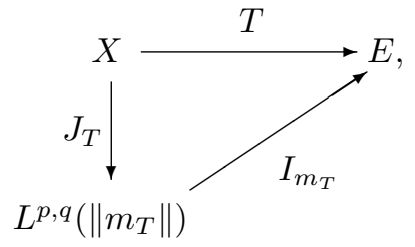

where $m_{T}: \Sigma \longrightarrow E$ is the vector measure associated to $T$. This section is devoted to study this kind of operators.

Remark 5.1. According to (2.3) we know that $L^{p, q}(\|m\|) \subseteq L^{p, p}(\|m\|) \subseteq L^{p}(m)$, for all $1 \leq q \leq p<\infty$, and these inclusions are in general strict. Thus, to be $(p, q)$-Lorentz factorable is stronger than being $p$-th power factorable.

In what follows we will need the following

Lemma 5.2. Let $m: \Sigma \longrightarrow E$ be a vector measure and let $\varphi$ be a simple function taking values $\alpha_{0}=0<\alpha_{1}<\cdots<\alpha_{n}$. Then, for $1 \leq p, q<\infty$,

$$
\|\varphi\|_{L^{p, q}(\|m\|)}^{q}=\frac{p}{q} \sum_{k=1}^{n}\left(\alpha_{k}^{q}-\alpha_{k-1}^{q}\right)\left(\|m\|\left(\left[\varphi \geq \alpha_{k}\right]\right)\right)^{\frac{q}{p}} .
$$

Proof. A simple computation shows that the distribution function with respect to $\|m\|$ of a simple function $\varphi$ taking values $\alpha_{0}:=0<\alpha_{1}<\cdots<\alpha_{n}$ is given by $\|m\|_{\varphi}=$ $\sum_{k=1}^{n}\|m\|\left(\left[\varphi \geq \alpha_{k}\right]\right) \chi_{\left[\alpha_{k-1}, \alpha_{k}\right)}$. Since $L^{p, q}(\|m\|)$ contains the simple functions, we can compute its quasi-norm according to (2.2) as

$$
\begin{aligned}
\|\varphi\|_{L^{p, q}(\|m\|)}^{q} & =p \int_{0}^{\infty} t^{q-1}\left(\|m\|_{\varphi}(t)\right)^{\frac{q}{p}} d t \\
& =p \int_{0}^{\infty} t^{q-1}\left(\sum_{k=1}^{n}\|m\|\left(\left[\varphi \geq \alpha_{k}\right]\right) \chi_{\left[\alpha_{k-1}, \alpha_{k}\right)}(t)\right)^{\frac{q}{p}} d t \\
& =p \int_{0}^{\infty} t^{q-1} \sum_{k=1}^{n}\left(\|m\|\left(\left[\varphi \geq \alpha_{k}\right]\right)\right)^{\frac{q}{p}} \chi_{\left[\alpha_{k-1}, \alpha_{k}\right)}(t) d t \\
& =p \sum_{k=1}^{n}\left(\|m\|\left(\left[\varphi \geq \alpha_{k}\right]\right)\right)^{\frac{q}{p}} \int_{\alpha_{k-1}}^{\alpha_{k}} t^{q-1} d t \\
& =\frac{p}{q} \sum_{k=1}^{n}\left(\alpha_{k}^{q}-\alpha_{k-1}^{q}\right)\left(\|m\|\left(\left[\varphi \geq \alpha_{k}\right]\right)\right)^{\frac{q}{p}} .
\end{aligned}
$$

Next theorem is the main result of this section and provides a characterization of the class of $(p, q)$-Lorentz factorable operators. In addition, it describes an optimality 
property of Lorentz spaces of the semivariation $L^{p, q}(\|m\|)$ associated to this class of operators.

Theorem 5.3. Let $X$ be an order continuous Banach function space over a finite measure $\mu$ and let $T: X \longrightarrow E$ be a Banach space valued operator. The following assertions are equivalent.

(i) $T$ is $(p, q)$-Lorentz factorable.

(ii) There is a constant $K>0$ such that

$$
\left(\sum_{k=1}^{n}\left(\alpha_{k}^{q}-\alpha_{k-1}^{q}\right)\left\|T\left(\chi_{B_{k} \cap\left[|f| \geq \alpha_{k}\right]}\right)\right\|_{E}^{\frac{q}{p}}\right)^{\frac{1}{q}} \leq K\|f\|_{X}
$$

for each $f \in X$ and each pair of finite sequences $\alpha_{0}:=0<\alpha_{1}<\cdots<\alpha_{n}$ and $B_{1}, \ldots, B_{n}$ in $\Sigma$, for all $n \in \mathbb{N}$.

Moreover, in this case the space $L^{p, q}\left(\left\|m_{T}\right\|\right)$ is optimal in the following sense: if there is another Banach function space $Z$ over $\mu$ such that $X \subseteq Z$ and $T$ can be extended as a $(p, q)$-Lorentz factorable operator $\tilde{T}: Z \longrightarrow E$, then $Z \subseteq L^{p, q}\left(\left\|m_{T}\right\|\right)$.

Proof. (ii) $\Rightarrow$ (i) By Lemma 5.2 we know that for a simple function $\varphi$ taking values $\alpha_{0}:=$ $0<\alpha_{1}<\cdots<\alpha_{n}$, its quasi-norm in the space $L^{p, q}\left(\left\|m_{T}\right\|\right)$ is given by

$$
\|\varphi\|_{L^{p, q}\left(\left\|m_{T}\right\|\right)}^{q}=\frac{p}{q} \sum_{k=1}^{n}\left(\alpha_{k}^{q}-\alpha_{k-1}^{q}\right)\left(\left\|m_{T}\right\|\left(\left[\varphi \geq \alpha_{k}\right]\right)\right)^{\frac{q}{p}} .
$$

Then

$$
\begin{aligned}
\|\varphi\|_{L^{p, q}\left(\left\|m_{T}\right\|\right)}^{q} & =\frac{p}{q} \sum_{k=1}^{n}\left(\alpha_{k}^{q}-\alpha_{k-1}^{q}\right)\left(\left\|m_{T}\right\|\left(\left[\varphi \geq \alpha_{k}\right]\right)\right)^{\frac{q}{p}} \\
& \leq \frac{p}{q} \sum_{k=1}^{n}\left(\alpha_{k}^{q}-\alpha_{k-1}^{q}\right) 2^{\frac{q}{p}} \sup _{B_{k} \in \Sigma}\left\|T\left(\chi_{B_{k} \cap\left[\varphi \geq \alpha_{k}\right]}\right)\right\|_{E}^{\frac{q}{p}} \\
& =\frac{p}{q} 2^{\frac{q}{p}} \sup _{B_{k} \in \Sigma} \sum_{k=1}^{n}\left(\alpha_{k}^{q}-\alpha_{k-1}^{q}\right)\left\|T\left(\chi_{B_{k} \cap\left[\varphi \geq \alpha_{k}\right]}\right)\right\|_{E}^{\frac{q}{p}} \leq \frac{p}{q} 2^{\frac{q}{p}} K^{q}\|\varphi\|_{X}^{q} .
\end{aligned}
$$

The last inequality follows from (5.2). Now, if $f \in X$, there exists a sequence $\left(\varphi_{n}\right)_{n}$ of simple function such that $0 \leq \varphi_{n} \uparrow|f|$ pointwise $\mu$-a.e. In particular $0 \leq \varphi_{n} \uparrow|f|$ pointwise $\left\|m_{T}\right\|$-a.e. and $\left\|\varphi_{n}\right\|_{L^{p, q}\left(\left\|m_{T}\right\|\right)} \leq\left(\frac{p}{q}\right)^{\frac{1}{q}} 2^{\frac{1}{p}} K\left\|\varphi_{n}\right\|_{X} \leq\left(\frac{p}{q}\right)^{\frac{1}{q}} 2^{\frac{1}{p}} K\|f\|_{X}$. Since $L^{p, q}\left(\left\|m_{T}\right\|\right)$ has the sequential Fatou property (see [3, Proposition 3.1]) we conclude that $\|f\|_{L^{p, q}\left(\left\|m_{T}\right\|\right)}=\sup _{n}\left\|\varphi_{n}\right\|_{L^{p, q}\left(\left\|m_{T}\right\|\right)} \leq\left(\frac{p}{q}\right)^{\frac{1}{q}} 2^{\frac{1}{p}} K\|f\|_{X}$, and $X \subseteq L^{p, q}\left(\left\|m_{T}\right\|\right)$. The commutativity of the diagram follows from the known facts that $L^{p, q}\left(\left\|m_{T}\right\|\right) \subseteq L^{1}\left(m_{T}\right)$, and $T(f)=\int_{\Omega} f d m_{T}$, for all $f \in X$.

(i) $\Rightarrow$ (ii) Take a function $f \in X$, a finite sequence $\alpha_{0}:=0<\alpha_{1}<\cdots<\alpha_{n}$, and a finite sequence $B_{1}, \ldots, B_{n}$ in $\Sigma$. From the inclusion $X \subseteq L^{p, q}\left(\left\|m_{T}\right\|\right)$ there exists a constant $M>0$ such that $\|f\|_{L^{p, q}\left(\left\|m_{T}\right\|\right)} \leq M\|f\|_{X}$. Consider the simple function

$$
\varphi:=\sum_{k=1}^{n} \alpha_{k-1} \chi_{\left[\alpha_{k}>|f| \geq \alpha_{k-1}\right]}+\alpha_{n} \chi_{\left[|f| \geq \alpha_{n}\right]} .
$$


Note that $0 \leq \varphi \leq|f|$ and $\left[|f| \geq \alpha_{k}\right]=\left[\varphi \geq \alpha_{k}\right]$ for all $k=1, \ldots, n$. Then

$$
\begin{aligned}
\sum_{k=1}^{n}\left(\alpha_{k}^{q}-\alpha_{k-1}^{q}\right)\left\|T\left(\chi_{B_{k} \cap\left[|f| \geq \alpha_{k}\right]}\right)\right\|_{E}^{\frac{q}{p}} & =\sum_{k=1}^{n}\left(\alpha_{k}^{q}-\alpha_{k-1}^{q}\right)\left\|T\left(\chi_{B_{k} \cap\left[\varphi \geq \alpha_{k}\right]}\right)\right\|_{E}^{\frac{q}{p}} \\
& =\sum_{k=1}^{n}\left(\alpha_{k}^{q}-\alpha_{k-1}^{q}\right)\left\|m_{T}\left(B_{k} \cap\left[\varphi \geq \alpha_{k}\right]\right)\right\|_{E}^{\frac{q}{p}} \\
& \leq \sum_{k=1}^{n}\left(\alpha_{k}^{q}-\alpha_{k-1}^{q}\right)\left(\left\|m_{T}\right\|\left(B_{k} \cap\left[\varphi \geq \alpha_{k}\right]\right)\right)^{\frac{q}{p}} \\
& \leq \sum_{k=1}^{n}\left(\alpha_{k}^{q}-\alpha_{k-1}^{q}\right)\left(\left\|m_{T}\right\|\left(\left[\varphi \geq \alpha_{k}\right]\right)\right)^{\frac{q}{p}} \\
& =\frac{q}{p}\|\varphi\|_{L^{p, q}\left(\left\|m_{T}\right\|\right)}^{q} \leq \frac{q}{p}\|f\|_{L^{p, q}\left(\left\|m_{T}\right\|\right)}^{q} \\
& \leq \frac{q}{p} M^{q}\|f\|_{X}^{q},
\end{aligned}
$$

as we wanted to prove.

Let us show now the optimality of the extension provided by $L^{p, q}\left(\left\|m_{T}\right\|\right)$. Suppose that $T$ can be extended to another Banach function space $Z$, with $X \subseteq Z$, as $\tilde{T}: Z \longrightarrow E$. Then we have that the vector measure $m_{\tilde{T}}: \Sigma \longrightarrow E$ associated to $\tilde{T}$ coincides with $m_{T}$ because $m_{\tilde{T}}(A)=\tilde{T}\left(\chi_{A}\right)=T\left(\chi_{A}\right)=m_{T}(A)$ for all $A \in \Sigma$. Since $\tilde{T}$ preserves the $(p, q)$-Lorentz factorability, we have that $Z \subseteq L^{p, q}\left(\left\|m_{\tilde{T}}\right\|\right)=L^{p, q}\left(\left\|m_{T}\right\|\right)$, as desired.

Remark 5.4. 1) It is not difficult to see that an operator $T: X \longrightarrow E$ is $(p, q)$-Lorentz factorable if and only if there is a constant $K>0$ such that

$$
\sum_{k=1}^{n}\left(\alpha_{k}-\alpha_{k-1}\right)\left\|T\left(\chi_{B_{k} \cap\left[|f| \geq \alpha_{k}\right]}\right)\right\|_{E}^{\frac{q}{p}} \leq K\left\||f|^{\frac{1}{q}}\right\|_{X}^{q}
$$

for each $f \in X$, each finite increasing sequence $\alpha_{0}:=0<\alpha_{1}<\cdots<\alpha_{n}$ and each finite sequence $B_{1}, \ldots, B_{n}$ in $\Sigma$. As we noted before, $X \subseteq X_{[q]}$, for $q \geq 1$, and consequently $|f|^{\frac{1}{q}} \in X$ for all $f \in X$. This inequality (5.3) can be compared with the definition of $q$-th power factorable operator given by the inequality (4.1).

2) Let us remark that if $E$ is a Banach lattice and $T$ is a positive operator, then

$$
\left\|T\left(\chi_{B \cap[|f| \geq \alpha]}\right)\right\|_{E} \leq\left\|T\left(\chi_{[|f| \geq \alpha]}\right)\right\|_{E}, \quad f \in X, \alpha>0, B \in \Sigma,
$$

and therefore the measurable sets $B_{k}$ can be dropped in the inequalities (5.2) and (5.3).

The $(p, q)$-Lorentz factorability of the identity map of a Banach function space $X$ over a finite measure $\mu$ defines a concavity-type lattice geometric property for Banach lattices of measurable functions. Let us finish this section by analyzing this property.

Definition 5.5. Let $1<p<\infty$ and $1 \leq q<\infty$ be. We say that a Banach function space $X$ of measurable functions over a finite measure $\mu$ is $(p, q)$-Lorentz concave if there is a constant $K>0$ such that $\sum_{k=1}^{n}\left(\alpha_{k}-\alpha_{k-1}\right)\left\|\chi_{\left[|f| \geq \alpha_{k}\right]}\right\|_{X}^{\frac{q}{p}} \leq K\left\||f|^{\frac{1}{q}}\right\|_{X}^{q}$, for every function $f \in X$ and each finite sequence $\alpha_{0}:=0<\alpha_{1}<\cdots<\alpha_{n}$. 
Remark 5.6. Note that Theorem 5.3 together with Remark 5.4 imply that an order continuous Banach function space is $(p, q)$-Lorentz concave if and only if the identity map in $X$ is $(p, q)$-Lorentz factorable.

Theorem 5.7. (Representation Theorem)

1) Let $m: \Sigma \longrightarrow E$ a vector measure. Then $L^{p, q}(\|m\|)$ is $(p, q)$-Lorentz concave.

2) If $X$ is a $(p, q)$-Lorentz concave order continuous Banach function space with a weak order unit, there is a Banach space valued vector measure $m: \Sigma \longrightarrow X$ such that $X$ is topologically and order isomorphic to $L^{p, q}(\|m\|)$.

Proof. 1) Consider a vector measure $m$ and take a function $f \in L^{p, q}(\|m\|)$, and a finite sequence $\alpha_{0}:=0<\alpha_{1}<\cdots<\alpha_{n}$. Consider the simple function

$$
\varphi:=\sum_{k=1}^{n} \alpha_{k-1} \chi_{\left[\alpha_{k}>|f| \geq \alpha_{k-1}\right]}+\alpha_{n} \chi_{\left[|f| \geq \alpha_{n}\right]} .
$$

Note that $0 \leq \varphi \leq|f|$ and $\left[|f| \geq \alpha_{k}\right]=\left[\varphi \geq \alpha_{k}\right]$ for all $k=1, \ldots, n$. Also, being $p>1$, we have the continuous inclusion $L^{p^{2}, q}(\|m\|) \subseteq L^{p, q}(\|m\|)$. Then

$$
\begin{aligned}
\sum_{k=1}^{n}\left(\alpha_{k}^{q}-\alpha_{k-1}^{q}\right)\left\|\chi_{\left[|f| \geq \alpha_{k}\right]}\right\|_{L^{p, q}(\|m\|)}^{\frac{q}{p}} & =\sum_{k=1}^{n}\left(\alpha_{k}^{q}-\alpha_{k-1}^{q}\right)\left\|\chi_{\left[\varphi \geq \alpha_{k}\right]}\right\|_{L^{p, q}(\|m\|)}^{\frac{q}{p}} \\
& =\sum_{k=1}^{n}\left(\alpha_{k}^{q}-\alpha_{k-1}^{q}\right)\left(\frac{p}{q}\right)^{\frac{1}{p}}\left(\|m\|\left(\left[\varphi \geq \alpha_{k}\right]\right)\right)^{\frac{q}{p^{2}}} \\
& =\left(\frac{p}{q}\right)^{\frac{1}{p}} \frac{q}{p^{2}} \frac{p^{2}}{q} \sum_{k=1}^{n}\left(\alpha_{k}^{q}-\alpha_{k-1}^{q}\right)\left(\|m\|\left(\left[\varphi \geq \alpha_{k}\right]\right)\right)^{\frac{q}{p^{2}}} \\
& =\left(\frac{p}{q}\right)^{\frac{1}{p}} \frac{q}{p^{2}}\|\varphi\|_{L^{p^{2}, q}(\|m\|)}^{q} \\
& \leq K\|\varphi\|_{L^{p, q}(\|m\|)}^{q} \leq K\|f\|_{L^{p, q}(\|m\|)}^{q} .
\end{aligned}
$$

This shows that the space $L^{p, q}(\|m\|)$ is $(p, q)$-Lorentz concave.

2) By $\left[4\right.$, Theorem 8] we know that $X$ is order and topologically isometric to $L^{1}(m)$ for a certain vector measure $m: \Sigma \longrightarrow X$. Moreover, this isometry is exactly the identity map and then it is $(p, q)$-Lorentz factorable, which means that $L^{1}(m) \subseteq L^{p, q}(\|m\|)$. But we know that the reverse inclusion $L^{p, q}(\|m\|) \subseteq L^{1}(m)$ always holds, and so the proof is over.

\section{Lattice GeOMEtRic PROPERTIES OF $L^{p, q}$ OF THE SEMIVARIATION}

Let us center now our attention in the lattice geometric properties of our class of spaces. According to the terminology for Banach lattices (see [17, 1.f.4] for example), a quasi-Banach lattice $X$ is said to satisfy an upper, respectively lower, p-estimate, for some $1 \leq p<\infty$, if there exists a constant $M>0$ such that, for every choice of pairwise disjoint elements $x_{1}, \ldots, x_{n}$ in $X$ we have

$$
\left\|\sum_{i=1}^{n} x_{i}\right\|^{p} \leq M \sum_{i=1}^{n}\left\|x_{i}\right\|^{p} \text {, respectively } \sum_{i=1}^{n}\left\|x_{i}\right\|^{p} \leq M\left\|\sum_{i=1}^{n} x_{i}\right\|^{p} .
$$

Let us note that if a quasi-Banach lattice is normable, that is, there exists a norm equivalent to the quasi-norm, then it satisfies an upper 1-estimate. In order to analyze when the Lorentz spaces $L^{p, q}(\|m\|)$ satisfy a lower estimate, we introduce the following 
terminology. We say that the semivariation $\|m\|$ of a vector measure $m$ has a lower $r$-estimate $(1 \leq r<\infty)$ if there exists $M>0$ such that

$$
\|m\|\left(A_{1} \cup \cdots \cup A_{n}\right) \geq M\left(\left(\|m\|\left(A_{1}\right)\right)^{r}+\cdots+\left(\|m\|\left(A_{n}\right)\right)^{r}\right)^{\frac{1}{r}}
$$

for every choice of pairwise disjoint sets $A_{1}, \ldots, A_{n}$ in $\Sigma$. Note that this condition implies that $m$ has finite $r$-variation. That is, we have that

$$
\sup \left\{\sum_{A \in \pi}\|m(A)\|_{E}^{r}: \pi \in \mathcal{P}_{f}(\Omega)\right\}<\infty,
$$

where $\mathcal{P}_{f}(\Omega)$ is the family of all finite partitions of $\Omega$ by means of measurable subsets.

Proposition 6.1. Let $m$ be a Banach space valued vector measure.

(i) If $1 \leq p \leq q<\infty$, then the space $L^{p, q}(\|m\|)$ has an upper p-estimate. In particular, it has an upper 1-estimate.

(ii) If $1 \leq q<p<\infty$, then the space $L^{p, q}(\|m\|)$ is a q-convex Banach lattice. In particular, it has an upper q-estimate.

(iii) If the semivariation has a lower $r$-estimate for some $r \geq 1$, then the space $L^{p, p r}(\|m\|)$ has a lower pr-estimate for each $1 \leq p<\infty$. In this case it is s-concave for each $s>p r$.

Proof. (i) Take a finite sequence of pairwise disjoint functions $f_{1}, \ldots, f_{n} \in L^{p, q}(\|m\|)$. Taking into account that $\left[\left|f_{1}+\cdots+f_{n}\right|>t\right]=\left[\left|f_{1}\right|>t\right] \cup \cdots \cup\left[\left|f_{n}\right|>t\right]$ for every $t>0$, we obtain $\|m\|_{f_{1}+\cdots+f_{n}}(t) \leq\|m\|_{f_{1}}(t)+\cdots+\|m\|_{f_{n}}(t)$, for each $t>0$. Now using the triangle inequality (for the norm of the weighted Lebesgue space $L^{\frac{q}{p}}\left(p t^{q-1} d t\right)$ ) we get

$$
\begin{aligned}
\left\|\sum_{i=1}^{n} f_{i}\right\|_{L^{p, q}(\|m\|)}^{p} & =\left(p \int_{0}^{\infty} t^{q-1}\left(\|m\|_{f_{1}+\cdots+f_{n}}(t)\right)^{\frac{q}{p}} d t\right)^{\frac{p}{q}} \\
& \leq \sum_{i=1}^{n}\left(p \int_{0}^{\infty} t^{q-1}\left(\|m\|_{f_{i}}(t)\right)^{\frac{q}{p}} d t\right)^{\frac{p}{q}}=\sum_{i=1}^{n}\left\|f_{i}\right\|_{L^{p, q}(\|m\|)^{p}}^{p}
\end{aligned}
$$

(ii) In this case $\frac{q}{p}<1$, and taking $\theta=1-\frac{q}{p}$ in Theorem 2.1 we have that

$$
L^{\frac{p}{q}, 1}(\|m\|)=\left(L^{1}(m), L^{\infty}(m)\right)_{\theta, 1} .
$$

Since $L^{1}(m)$ and $L^{\infty}(m)$ are Banach spaces, the real interpolated space $L^{\frac{p}{q}, 1}(\|m\|)$ is a normable (equivalently 1-convex), quasi-Banach lattice. Thus, its $q$-convexification $L^{p, q}(\|m\|)$ is $q$-convex.

(iii) Let us assume that the semivariation $\|m\|$ of $m$ has a lower $r$-estimate for $r \geq 1$. Take a finite pairwise disjoint sequence $f_{1}, \ldots, f_{n} \in L^{p, p r}(\|m\|)$. Then

$$
\begin{aligned}
\sum_{i=1}^{n}\left\|f_{i}\right\|_{L^{p, p r}}^{p r}(\|m\|) & =\sum_{i=1}^{n} p \int_{0}^{\infty} t^{p r-1}\left(\|m\|_{f_{i}}(t)\right)^{r} d t=p \int_{0}^{\infty} t^{p r-1} \sum_{i=1}^{n}\left(\|m\|_{f_{i}}(t)\right)^{r} d t \\
& =p \int_{0}^{\infty} t^{p r-1} \sum_{i=1}^{n}\left(\|m\|\left(\left[\left|f_{i}\right|>t\right]\right)\right)^{r} d t \\
& \leq \frac{p}{M^{r}} \int_{0}^{\infty} t^{p r-1}\left(\|m\|\left(\left[\left|f_{1}\right|>t\right] \cup \cdots \cup\left[\left|f_{n}\right|>t\right]\right)\right)^{r} d t \\
& =\frac{p}{M^{r}} \int_{0}^{\infty} t^{p r-1}\left(\|m\|_{f_{1}+\cdots+f_{n}}(t)\right)^{r} d t=\frac{1}{M^{r}}\left\|\sum_{i=1}^{n} f_{i}\right\|_{L^{p, p r}(\|m\|)}^{p r}
\end{aligned}
$$


The second statement follows from a result by Maurey and Pisier (see for instance [17, 1.f.7]).

Remark 6.2. In connection with Remark 4.2, if in addition we suppose there that the semivariation $\left\|m_{\theta, q}\right\|$ also has a lower $\frac{q}{r}$-estimate for some $q \geq r$, then Proposition 6.1 tells us that the interpolated operator $T_{\theta, q}$ can be extended to a reflexive Banach function space that has an upper $r$-estimate and a lower $q$-estimate, that is, the space $L^{r, q}\left(\left\|m_{\theta, q}\right\|\right)$.

\section{Final EXAmples AND APPLiCATiOnS}

In order to show some applications of our technique in its natural context, we will show now some results on operators from different classical Lebesgue weighted $L^{p}$-spaces and Lorentz spaces. Let us apply again Corollary 3.3 to another class of operators. Consider a Banach function space $X$ over a finite measure $\mu$. For $1 \leq p, q<\infty$, a $\mu$-determined operator $T: X \longrightarrow E$, with values into a Banach space $E$, is said to be bidual $(p, q)$-power-concave if there exists a weight $0<w \in L^{1}(\mu)$ such that the inclusions $X \subseteq L^{q}(w d \mu) \subseteq L^{p}\left(m_{T}\right)$ are continuous. Here $L^{q}(w d \mu)$ denotes the Lebesgue $L^{q}$-space for the finite measure with density $w$ given by $A \mapsto \int_{A} w d \mu$ (see [20, Theorem 6.9] for other characterizations of such operators). Bidual $(1, q)$-power-concave operators are of particular relevance. They are known also as bidual q-concave operators. Recall that a bidual $q$-concave operator is, in particular, $q$-concave (see [12, Proposition 6.2 (i)] with $p=1)$.

Proposition 7.1. Suppose that $T$ is an admissible $\mu$-determined operator between the couples $\left(X_{0}, X_{1}\right)$ and $\left(E_{0}, E_{1}\right)$ such that the restrictions $T_{0}$ and $T_{1}$ are bidual $(p, q)$-powerconcave for some $1 \leq p<\infty$ and $1 \leq q<\infty$. Then for each $0<\theta<1$ the interpolated operator $T_{\theta, q}:\left(X_{0}, X_{1}\right)_{\theta, q} \longrightarrow\left(E_{0}, E_{1}\right)_{\theta, q}$ is bidual $(p, q)$-power-concave.

Proof. Since $T_{0}$ and $T_{1}$ are bidual $(p, q)$-power-concave, there exist two weights $w_{0}>0$ and $w_{1}>0$ in $L^{1}(\mu)$ such that $X_{0} \subseteq L^{q}\left(w_{0} d \mu\right) \subseteq L^{p}\left(m_{0}\right)$ and $X_{1} \subseteq L^{q}\left(w_{1} d \mu\right) \subseteq$ $L^{p}\left(m_{1}\right)$. Then we have the inclusions

$$
\left(X_{0}, X_{1}\right)_{\theta, q} \subseteq\left(L^{q}\left(w_{0} d \mu\right), L^{q}\left(w_{1} d \mu\right)\right)_{\theta, q} \subseteq\left(L^{p}\left(m_{0}\right), L^{p}\left(m_{1}\right)\right)_{\theta, q} .
$$

Now, by the Stein-Weiss's interpolation theorem (see [23] or [1, Theorem 5.4.1]) the following equality $\left(L^{q}\left(w_{0} d \mu\right), L^{q}\left(w_{1} d \mu\right)\right)_{\theta, q}=L^{q}\left(w_{0}^{1-\theta} w_{1}^{\theta} d \mu\right)$ holds with equivalence of norms. From Corollary 3.3 we know that $\left(L^{p}\left(m_{0}\right), L^{p}\left(m_{1}\right)\right)_{\theta, q} \subseteq L^{p}\left(m_{\theta, q}\right)$, and then $\left(X_{0}, X_{1}\right)_{\theta, q} \subseteq L^{q}\left(w_{0}^{1-\theta} w_{1}^{\theta} d \mu\right) \subseteq L^{p}\left(m_{\theta, q}\right)$ as we wanted to prove.

Corollary 7.2. Let $\left(X_{0}, X_{1}\right)$ be a couple of q-convex order continuous B.f.s. and $T$ an admissible operator. If $T_{0}$ and $T_{1}$ are $q$-concave, then $T_{\theta, q}$ is $q$-concave for all $0<\theta<1$.

Proof. Since $X_{0}$ and $X_{1}$ are $q$-convex and $T_{0}$ and $T_{1}$ are $q$-concave, then applying [20, Proposition 6.2 (iv) and (6.6)] it follows that $T_{0}$ and $T_{1}$ are bidual $q$-concave operators. Then so is $T_{\theta, q}$, by Proposition 7.1 with $p=1$. Thus, [20, Proposition 6.2 (i)] guarantees that $T_{\theta, q}$ is $q$-concave.

The real interpolation spaces $\left(L^{r_{0}}\left(w_{0} d \mu\right), L^{r_{1}}\left(w_{1} d \mu\right)\right)_{\theta, q}$, with different weights $0<$ $w_{0}, w_{1} \in L^{1}(\mu)$, have been extensively treated in the literature in the last fifty years for different cases depending on the parameters $0<\theta<1 \leq q<\infty$, and $1 \leq r_{0}, r_{1}<\infty$. The obtained results depend on whether $r_{0}$ and $r_{1}$ are equal or not, and if $\frac{1}{q}=\frac{1-\theta}{r_{0}}+\frac{\theta}{r_{1}}$ 
(the diagonal case) or $\frac{1}{q} \neq \frac{1-\theta}{r_{0}}+\frac{\theta}{r_{1}}$ (the off-diagonal case). In the diagonal case, the classical real interpolation result of Peetre (see [1, Theorem 5.5.1]) states the wellknown formula $\left(L^{r_{0}}\left(w_{0} d \mu\right), L^{r_{1}}\left(w_{1} d \mu\right)\right)_{\theta, q}=L^{q}\left(w_{0}^{1-\theta} w_{1}^{\theta} d \mu\right)$. In the off-diagonal case, the equation that is known as the Lizorkin-Freitag formula describes, for $r_{0} \neq r_{1}$, the interpolation space $\left(L^{r_{0}}\left(w_{0} d \mu\right), L^{r_{1}}\left(w_{1} d \mu\right)\right)_{\theta, q}$ as a weighted Lorentz space associated to a certain measure (see $[11,18]$ for the details). In the off-diagonal case with $r=r_{0}=r_{1}$, the interpolation spaces are related to Beurling-Hertz's spaces and have been described by Peetre and Gilbert as unions, if $r>q$, or intersections, if $r<q$, of weighted $L^{r}$ spaces, see [15, 21]. See also Persson [22] for spaces of vector-valued functions. However, in the general case there is not a satisfactory description of the interpolated space $\left(L^{r_{0}}\left(w_{0} d \mu\right), L^{r_{1}}\left(w_{1} d \mu\right)\right)_{\theta, q}$.

Now suppose that $T: L^{r_{0}}\left(w_{0} d \mu\right)+L^{r_{1}}\left(w_{1} d \mu\right) \longrightarrow E$ is an admissible $\mu$-determined operator with values into a Banach space $E$. Denote by $m$ its associated vector measure. Assume that the restrictions $T_{0}$ and $T_{1}$ are $p_{0}$-th and $p_{1}$-th power factorable respectively, with $1<p_{0} \neq p_{1}<\infty$. Note that being $p$-th power factorable depends strongly on the weights. Then Theorem 4.3 tells us that the interpolated operator $T$ : $\left(L^{r_{0}}\left(w_{0} d \mu\right), L^{r_{1}}\left(w_{1} d \mu\right)\right)_{\theta, q} \longrightarrow E$ is $(p, q)$-Lorentz factorable for $\frac{1}{p}=\frac{1-\theta}{p_{0}}+\frac{\theta}{p_{1}}$, which means that $\left(L^{r_{0}}\left(w_{0} d \mu\right), L^{r_{1}}\left(w_{1} d \mu\right)\right)_{\theta, q} \subseteq L^{p, q}(\|m\|)$ continuously. This inclusion, together with the lattice geometrical properties of the space $L^{p, q}(\|m\|)$ obtained in Section 6 , provide additional information about the interpolation space $\left(L^{r_{0}}\left(w_{0} d \mu\right), L^{r_{1}}\left(w_{1} d \mu\right)\right)_{\theta, q}$.

Finally we analyze the properties of the convolution operators defined by measures on topological groups, giving more information about the geometric and topological properties of some Banach lattices through which convolution operators given by $L^{q}$ improving measures can be factored. These operators provide a source of examples of canonical $p$-th power factorable operators.

Consider a regular Borel probability measure $\lambda$ on an Abelian compact topological group $G$. Let $1 \leq r<\infty$ and consider the convolution operator

$$
C: f \in L^{r}(G) \longrightarrow C(f):=f * \lambda \in L^{r}(G),
$$

where $f * \lambda(x):=\int_{G} f(x-y) d \lambda(y)$ for all $x \in G$. See [20, Chapter 7] for more information. Recall that for the classical Lorentz spaces, we have that $\left(L^{r_{0}}(G), L^{r_{1}}(G)\right)_{\theta, q}=L^{r, q}(G)$, where $\frac{1}{r}=\frac{1-\theta}{r_{0}}+\frac{\theta}{r_{1}}$, and $0<\theta<1 \leq q<\infty$. Moreover, the restriction $C: L^{r, q}(G) \longrightarrow$ $L^{r, q}(G)$ of the convolution operator to the Lorentz space $L^{r, q}(G)$ is continuous. Let us denote by $m_{r, q}$ the vector measure associated to the restriction to $L^{r, q}(G)$ of the convolution operator.

The measure $\lambda$ is said to be $L^{q}$-improving, for some $1 \leq q<\infty$, if there is $r \in(q, \infty)$ such that $\lambda * f \in L^{r}(G)$ for every $f \in L^{q}(G)$ (see [16]). In other words, the corresponding convolution operator has a smaller range than the one that is originally given by the measure $\lambda$. The following corollary (see [20, Corollary 7.103]), that is known for $L^{q}$ improving measures, is the key of our results.

Corollary 7.3. With the above notation, the following statements are equivalent.

(i) $\lambda$ is an $L^{q}$-improving measure.

(ii) For each $1<r<\infty$, the convolution operator $C: L^{r}(G) \longrightarrow L^{r}(G)$ is $p$-th power factorable for some $p \in(1, \infty)$. 
Let us assume that the convolution operators $C: L^{r_{0}}(G) \longrightarrow L^{r_{0}}(G)$ and $C$ : $L^{r_{1}}(G) \longrightarrow L^{r_{1}}(G)$ are $p$-th power factorable for some $1<p<\infty$. Note that, if an operator is $p$-th power factorable, then it is $s$-th power factorable for every $1 \leq s \leq p$. According to Remark 4.2, for each $0<\theta<1 \leq q<\infty$, the operator $C: L^{r, q}(G) \longrightarrow L^{r, q}(G)$, where $\frac{1}{r}=\frac{1-\theta}{r_{0}}+\frac{\theta}{r_{1}}$, factors through the Lorentz space $L^{s, q}\left(\left\|m_{r, q}\right\|\right)$, for each $1 \leq s<p$, as

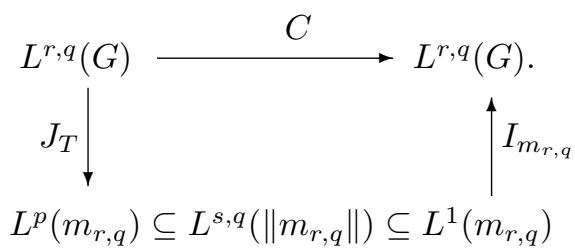

Under some further requirements, the properties of the factorization space $L^{s, q}\left(\left\|m_{r, q}\right\|\right)$ that have been explained in Proposition 6.1 provide the following result.

Corollary 7.4. Assume that the requirements in (any of) the statements (i) and (ii) of Corollary 7.3 hold. Suppose in addition that the semivariation $\left\|m_{r, q}\right\|$ has a lower $\frac{q}{s}$-estimate. Then the convolution operator $C: L^{r, q}(G) \longrightarrow L^{r, q}(G)$ can be extended to a reflexive Banach function space that has an upper s-estimate and a lower q-estimate.

\section{REFERENCES}

1. J. Bergh and J. Löfström, Interpolation Spaces. Springer-Verlag, Berlin New York, 1976.

2. R. del Campo, A. Fernández, O. Galdames, F. Mayoral and F. Naranjo, Complex interpolation of operators and optimal domains, Integr. Equ. Oper. Theory 80 (2014), 229-238.

3. R. del Campo, A. Fernández, F. Mayoral and F. Naranjo, Reflexivity of function spaces associated to a $\sigma$-finite vector measure, J. Math. Anal. Appl. 438 (2016), 339-350.

4. G. P. Curbera, Operators into $L^{1}$ of a vector measure and applications to Banach lattices, Math. Ann. 293 (1992) 317-330.

5. G. P. Curbera and W. J. Ricker, Optimal domains for kernel operators via interpolation, Math. Nachr. 244 (2002), 47-63.

6. M. Cwikel, P. G. Nilsson and G. Schechtman, Interpolation of weighted Banach lattices. A characterization of relatively decomposable Banach lattices, Mem. Amer. Math. Soc. 165 (2003), no. 787 .

7. A. Defant, Variants of the Maurey-Rosental theorem for quasi Köthe function spaces, Positivity $\mathbf{5}$ (2001), 153-175.

8. J. Diestel and J. J. Uhl, Vector Measures. Math. Surveys, vol. 15, Amer. Math. Soc., Providence, R.I., 1977.

9. A. Fernández, F. Mayoral and F. Naranjo, Real interpolation method on spaces of scalar integrable functions with respect to vector measures, J. Math. Anal. Appl. 376 (2011), 203-211.

10. A. Fernández, F. Mayoral, F. Naranjo, C. Sáez and E. A. Sánchez-Pérez, Spaces of p-integrable functions with respect to a vector measure, Positivity 10 (2006), 1-16.

11. D. Freitag, Real interpolation of weighted $L_{p}$-spaces, Math. Nachr. 86 (1978), 15-18.

12. O. Galdames Bravo, Duality theory for $p$-th power factorable operators and kernel operators, Doctoral Thesis, Universitat Politècnica de València, 2013.

13. O. Galdames Bravo and E.A. Sánchez Pérez, Optimal range theorems for operators with p-th power factorable adjoints, Banach J. Math. Anal. 6,1 (2012), 61-73.

14. O. Galdames Bravo and E.A. Sánchez Pérez, Factorizing kernel operators, Integr. Equ. Oper. Theory 75 (2013), 13-29.

15. J. E. Gilbert, Interpolation between weighted $L^{p}$-spaces, Ark. Math. 10 (1972), 235-249.

16. K. H. Hare, A characterization of $L^{p}$-improving measures, Proc. Amer. Math. Soc. 102, no. 2, (1988), 295-299.

17. J. Lindenstrauss and L. Tzafriri, Classical Banach Spaces II. Springer-Verlag, Berlin, 1979.

18. P. I. Lizorkin, Interpolation of $L_{p}$-spaces with a weight, Trudy Mat. Inst. Steklov. 140 (1976), 201-211 (in Russian); English transl.: Proc. Steklov Inst. Math. 140 (1979), 221-232. 
19. L. Maligranda, The K-functional for p-convexifications, Positivity 17 (2013), no. 3, 707-710.

20. S. Okada, W. J. Ricker and E. A. Sánchez Pérez, Optimal Domain and Integral Extension of Operators acting in Function Spaces, Operator Theory: Adv. Appl., vol. 180, Birkhäuser, Basel, 2008.

21. J. Peetre, On interpolation of $L^{p}$-spaces with weight functions, Acta Sci. Math. (Szeged) 28 (1967), 61-69.

22. L. E. Persson, Descriptions of some interpolation spaces in off-diagonal cases, in: Interpolation spaces and allied topics in analysis, Lund 1983, 213-231, Lecture Notes in Math. 1070, SpringerVerlag, 1984.

23. E. M. Stein and G. Weiss, Interpolation of operators with change of measures, Trans. Amer. Math. Soc. 87 (1958), 159-172.

R. del Campo, Departamento de Matemática Aplicada I,

EUITA, Universidad de Sevilla,

Ctra. de Utrera Km. 1, 41013, Sevilla. Spain.

Email address: rcampo@us.es

A. Fernández, Departamento de Matemática Aplicada II,

Escuela Técnica Superior de Ingeniería, Universidad de Sevilla,

Camino de los Descubrimientos s/n, 41092, Sevilla. Spain.

Email address: afcarrion@us.es

F. Mayoral, Departamento de Matemática Aplicada II,

Escuela Técnica Superior de Ingeniería, Universidad de Sevilla,

Camino de los Descubrimientos S/n, 41092, Sevilla. Spain.

Email address: mayoral@us.es

F. Naranjo, Departamento de Matemática Aplicada II,

Escuela Técnica Superior de Ingeniería, Universidad de Sevilla,

Camino de los Descubrimientos S/n, 41092, Sevilla. Spain.

Email address: naranjo@us.es

E.A. SÁnchez Pérez, Instituto Universitario de Matemática Pura y Aplicada, Universitat Politècnica de ValÈncia,

Camino de Vera s/n, 46022, Valencia. Spain.

Email address: easancpe@mat.upv.es 